\title{
Validation study of the modified injection technique for internal mammary sentinel lymph node biopsy in breast cancer
}

This article was published in the following Dove Press journal:

OncoTargets and Therapy

24 September 2015

Number of times this article has been viewed

\author{
Bin-Bin Cong ${ }^{1,2, *}$ \\ Xiao-Shan $\mathrm{Cao}^{1,2, *}$ \\ Peng-Fei Qiu' \\ Yan-Bing Liu' \\ Tong Zhao' \\ Peng Chen' \\ Chun-Jian Wang' \\ Zhao-Peng Zhang' \\ Xiao Sun' \\ Yong-Sheng Wang' \\ 'Breast Cancer Center, Shandong \\ Cancer Hospital and Institute, ${ }^{2} \mathrm{School}$ \\ of Medicine and Life Sciences, Jinan \\ University-Shandong Academy of \\ Medical Sciences, Jinan, Shandong, \\ People's Republic of China \\ *These authors contributed equally \\ to this study
}

Correspondence: Yong-Sheng Wang Breast Cancer Center, Shandong Cancer Hospital and Institute, No 440, Jiyan Road, Jinan, Shandong 250II7, People's Republic of China Email wangysh2008@aliyun.com

\begin{abstract}
According to the hypothesis of internal mammary sentinel lymph node (IM-SLN) lymphatic drainage pattern, a modified radiotracer injection technique (periareolar intraparenchyma, high volume, and ultrasonographic guidance) was established. To verify the accuracy of the hypothesis and validate the modified radiotracer injection technique and to observe whether the lymphatic drainage of the whole breast parenchyma could reach to the same IMSLN, different tracers were injected into different locations of the breast. The validation study results showed that the correlation and the agreement of the radiotracer and the fluorescence tracer are significant (case-base, $r_{\mathrm{s}}=0.808, P<0.001$; Kappa $=0.79, P<0.001$ ). It proved that the lymphatic drainage from different location of the breast (the primary tumor, the subareolar plexus) reached the same IM-SLNs and the hypothesis of IM-SLN lymphatic drainage pattern (ie, IM-SLN receives lymphatic drainage from not only the primary tumor area, but also the entire breast parenchyma). In other words, it validated the accuracy of our modified radiotracer injection technique.
\end{abstract}

Keywords: breast cancer, internal mammary, sentinel lymph node biopsy, visualization rate

\section{Introduction}

Cao et $\mathrm{al}^{1}$ have emphasized the importance of internal mammary sentinel lymph node biopsy (IM-SLNB) and have described a modified radiotracer injection technique that could significantly improve the visualization rate of the internal mammary sentinel lymph node (IM-SLN). Even though the TNM staging of the sixth American Joint Committee on Cancer incorporated the concept of IM-SLNB, IM-SLNB is not routinely performed and remains a subject of discussion. One reason is the low visualization rate of IM-SLN with the traditional radiotracer injection technique (range from $0 \%$ to $37 \%$, mean $13 \%) .^{2-4}$ The other reason is previous IM-SLNB clinical trials failed to evaluate the status of internal mammary lymph node (IMLN) in patients who were really in need (only in patients with clinically negative axillary lymph node [ALN]), because IMLN metastases were mostly found concomitantly with ALN metastases.

\section{Modified radiotracer injection technique}

Current evidence has proved that the axillary sentinel lymph node (ASLN) receives the lymphatic drainage from not only the primary tumor area but also the entire breast organ. ${ }^{5}$ Tanis et al described that the breast parenchyma has extensive lymphatic network and has rich anastomoses with the superficial cutaneous lymph plexus of the developing skin. ${ }^{6}$ It is considered that radiotracer, wherever injected, could flow to the same ASLN. 
Based on the earlier concept of ASLN, we hypothesized that the IM-SLN received lymphatic drainage from not only the primary tumor area but also the entire breast parenchyma (in other words, the radiotracer could flow to the same IM-SLN wherever injected). According to the hypothesis of IM-SLN lymphatic drainage pattern, a modified radiotracer injection technique (periareolar intraparenchyma, high volume, and ultrasonographic guidance) was established. ${ }^{7}$ In comparison with the traditional radiotracer injection technique, the modified radiotracer injection technique significantly enhanced the visualization rate of IM-SLN without lowering the ASLN visualization rate $(99.4 \%, 155 / 156$ in the validation study), thus providing the technical feasibility for IM-SLNB and improving the pathological stage.

\section{Validation procedure}

The accuracy of axillary sentinel lymph node biopsy (SLNB) has been proved by subsequent axillary lymph node dissection. However, the accuracy of IM-SLNB has not been confirmed. As the extended radical mastectomy has been abandoned since $1960 \mathrm{~s},{ }^{8}$ we cannot validate the accuracy of IM-SLNB and the modified technology by this way. Recently, a validation study about the accuracy of the modified technique was designed and performed by our team in our breast cancer center. The study was approved by the Shandong Cancer Hospital and Institute Ethics Committee (No SDTHEC20130324). Written, informed consent was obtained from all patients. In the validation study, the indocyanine green (ICG) fluorescence tracer, which is a safe and effective method for SLNB in breast cancer with acceptable sensitivity and specificity comparable to conventional methods (blue dye and radiotracer), ${ }^{9-11}$ and the radiotracer were injected into different locations of the breast to observe whether the lymphatic drainage of the whole breast parenchyma could reach the same IM-SLN.

A total of 156 patients with breast cancer were enrolled in this study from September 2013 to December 2014. The radiotracer ${ }^{99 \mathrm{~m}} \mathrm{Tc}-$ labeled sulfur colloid, $1.0-1.2 \mathrm{~mL}$, 9.25-18.5 MBq) was injected with our modified radiotracer injection technique, and the injection sites were chosen at the 6 and 12 o'clock positions, $0.5-1.0 \mathrm{~cm}$ from the areola, under ultrasonographic guidance 3-18 hours before surgery. ${ }^{7}$ All patients underwent a preoperative lymphoscintigraphy (Toshiba GCA 901AHG; Toshiba, Tokyo, Japan) 30 minutes before surgery and a gamma probe (Neoprobe, Neo2000 gamma detection system; Johnson \& Johnson New Brunswick, NJ, USA) detection 10 minutes before surgery. Radioactive IM-SLNs was defined as IM-SLNs visualized on preoperative lymphoscintigraphy (Figure 1) and detected

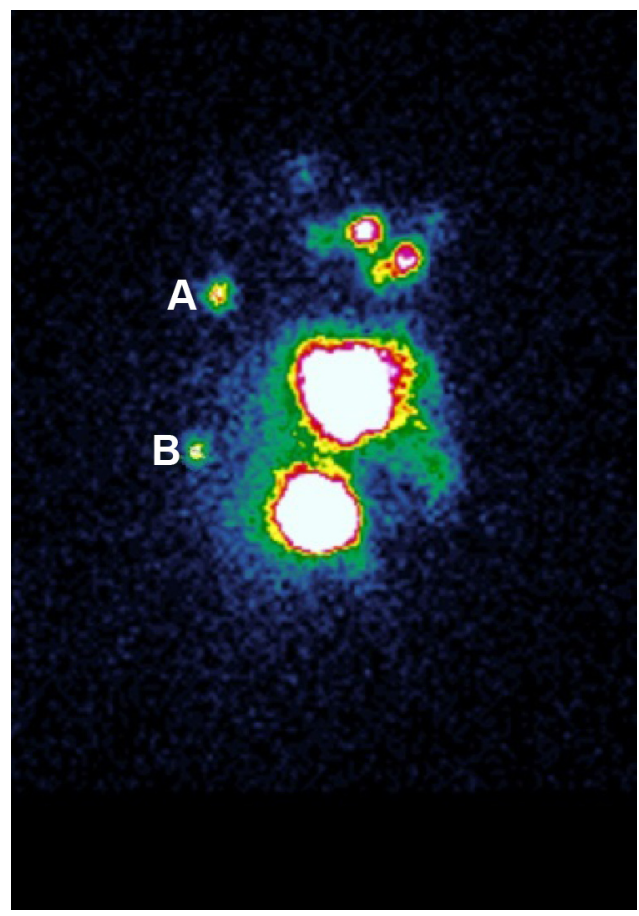

Figure I Lymphoscintigram 30 minutes before surgery.

Note: Hotspots (A and $\mathbf{B}$ ) are evidently shown in both the second intercostal space (A) and the fourth intercostal space (B) in patient with left-sided breast cancer.

by gamma probe. Patients with radioactive IM-SLNs were injected with ICG 5 minutes before surgery. A total of $2 \mathrm{~mL}$ $0.5 \%$ ICG was intraparenchymally injected under the ultrasonographic guidance at the peritumoral near the subareolar region to differentiate it from the radiotracer. IM-SLNB was performed for patients with the radioactive IM-SLNs. The fluorescent signal of IM-SLN was identified by the fluorescence imaging system (Mingde, MD fluorescence imaging system, Langfang, People's Republic of China; Figure 2). The flowchart of the validation study is shown in Figure 3.

In this study, IM-SLNs were visualized on preoperative lymphoscintigraphy and detected by gamma probe in 112 cases, and the IM-SLN visualization rate was $71.8 \%$ $(112 / 156)$. A total of 110 of them successfully underwent IM-SLNB, so the success rate of IM-SLNB was $98.2 \%$ $(110 / 112)$. There were 94 patients with the IM-SLN verified both radioactive and fluorescence tracer positive, and 16 patients with the IM-SLN only verified radiotracer positive. The other 44 cases found no IM-SLN with either radioactive or fluorescence tracer positive. The results showed that the correlation and the agreement of the radiotracer and the fluorescence tracer are significant (case-base, $r_{\mathrm{s}}=0.808$, $P<0.001$; Kappa $=0.79, P<0.001$ ). The median number of IM-SLNs was 2 (total 198, range: 1-4 nodes). The IM-SLNs were located in the first $(6.0 \%, 12 / 198)$, second $(46.5 \%, 92 / 198)$, third $(39.9 \%, 79 / 198)$ and forth $(7.6 \%$, 

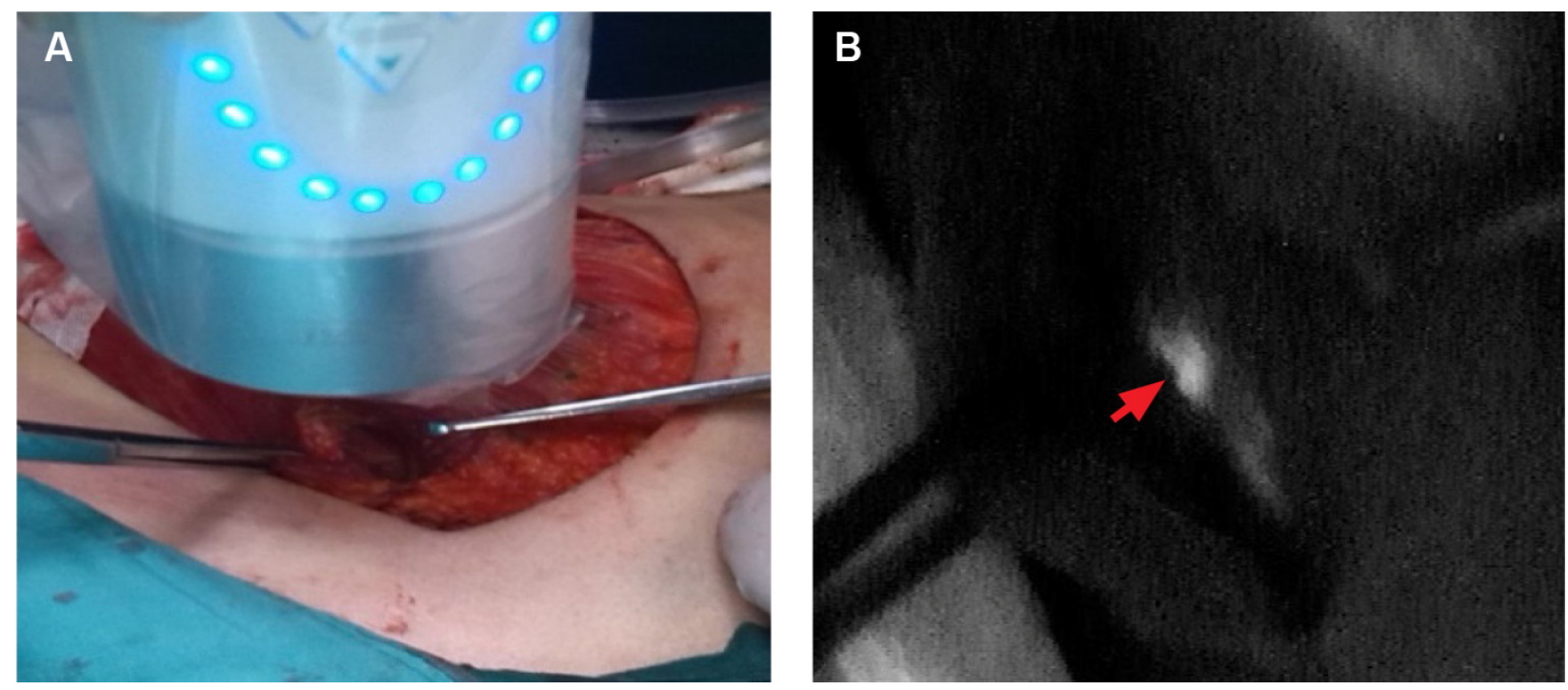

Figure 2 Intraoperatively IM-SLNB via intercostal space.

Note: The fluorescence imaging system (A) showed an IM-SLN fluorescence tracer positive in the fourth intercostal space (arrow) in patient after mastectomy (B). Abbreviations: IM-SLN, internal mammary sentinel lymph node; IM-SLNB, internal mammary sentinel lymph node biopsy.

15/198) intercostal space, all positive IM-SLNs were in the second $(64.7 \%, 11 / 17)$ and third $(35.3 \%, 6 / 17)$ intercostal space. The involvement rate of IM-SLN was $6.8 \%(5 / 73)$ in patients with clinically negative ALN and $21.6 \%$ (8/37) in patients with clinically positive ALN. These results were in accordance with past studies of extended radical mastectomy. ${ }^{12}$ It can indirectly reflect the accuracy of our results. In six patients, the nodal pathological staging altered from $\mathrm{pN}_{2 \mathrm{a}}$ to $\mathrm{pN}_{3 \mathrm{~b}}$, causing a stage migration from IIIA to IIIC. Moreover, in five patients, the nodal pathological status changed from $\mathrm{pN}_{0}$ to $\mathrm{pN}_{1 \mathrm{~b}}$, resulting in the change of stage from IIA to IIB.

\section{Conclusion}

The results of our validation study showed that the lymphatic drainage from different locations of the breast (the primary tumor, the subareolar plexus) reached to the same IM-SLNs. On the one hand, it is proved that the nodes that are detected by IM-SLNB could drain not only the regional of the primary tumor but also the entire mammary glands. On the other hand, it is proved that the IM-SLN that was removed by IM-SLNB should be the "true" sentinel nodes. In conclusion, our study provided a factual basis to clarify the concept of IM-SLN and validated the accuracy of IM-SLNB and our modified radiotracer injection technique.

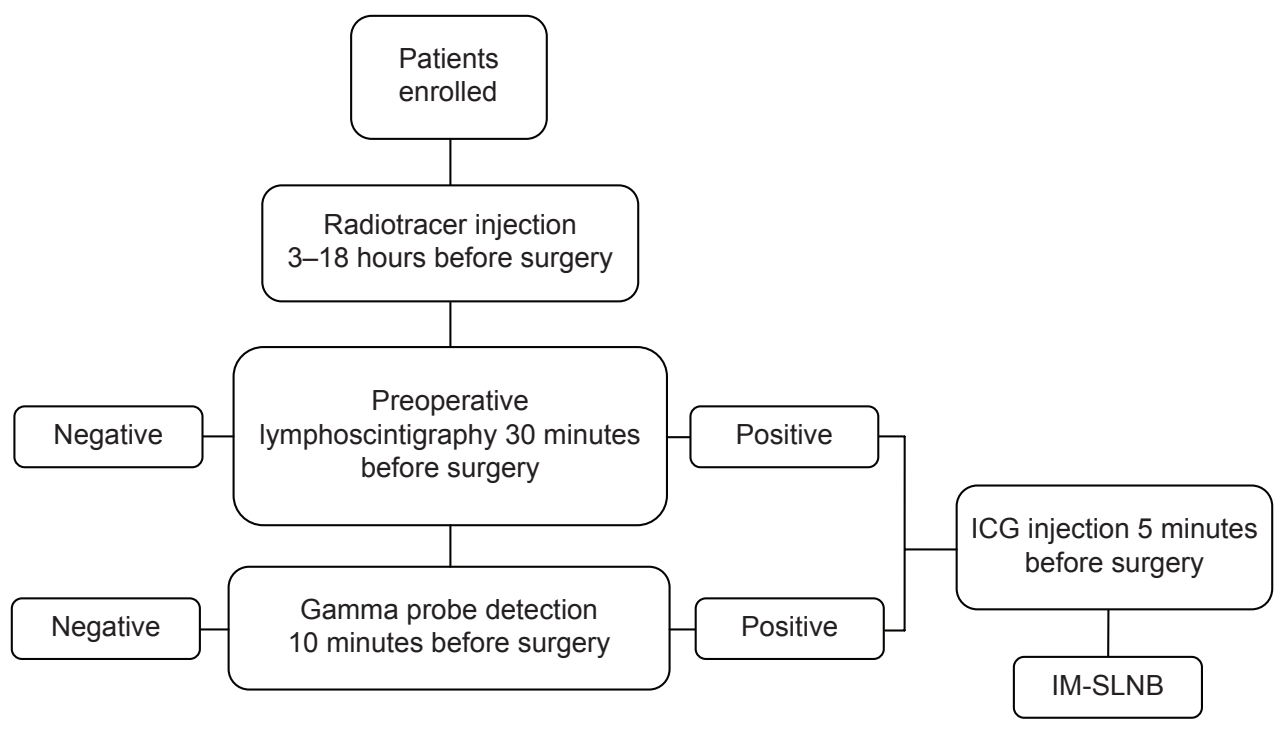

Figure 3 Flowchart of the validation study.

Abbreviations: ICG, indocyanine green; IM-SLNB, internal mammary sentinel lymph node biopsy. 


\section{Disclosure}

The authors report no conflicts of interest in this work.

\section{References}

1. Cao X, Wang C, Liu Y, Qiu P, Cong B, Wang Y. Axillary and internal mammary sentinel lymph node biopsy in male breast cancer patients: case series and review. Onco Targets Therapy. 2015;8:1499-1502.

2. Postma EL, van Wieringen S, Hobbelink MG, et al. Sentinel lymph node biopsy of the internal mammary chain in breast cancer. Breast Cancer Res Treat. 2012;134(2):735-741.

3. Heuts EM, van der Ent FW, von Meyenfeldt MF, Voogd AC. Internal mammary lymph drainage and sentinel node biopsy in breast cancer - A study on 1,008 patients. Eur J Surg Oncol. 2009;35(3):252-257.

4. Coombs NJ, Boyages J, French JR, Ung OA. Internal mammary sentinel nodes: ignore, irradiate or operate? Eur J Cancer. 2009;45(5): 789-794.

5. Borgstein PJ, Meijer S, Pijpers RJ, van Diest PJ. Functional lymphatic anatomy for sentinel node biopsy in breast cancer: echoes from the past and the periareolar blue method. Ann Surg. 2000;232(1):81-89.

6. Tanis PJ, Nieweg OE, Valdés Olmos RA, Kroon BB. Anatomy and physiology of lymphatic drainage of the breast from the perspective of sentinel node biopsy. J Am Coll Surg. 2001;192(3):399-409.
7. Qiu PF, Liu JJ, Liu YB, Yang GR, Sun X, Wang YS. A modified technology could significantly improve the visualization rate of the internal mammary sentinel lymph nodes in breast cancer patients. Breast Cancer Res Treat. 2012;136(1):319-321.

8. Cong BB, Qiu PF, Wang YS. Internal mammary sentinel lymph node biopsy: minimally invasive staging and tailored internal mammary radiotherapy. Ann Surg Oncol. 2014;21(7):2119-2121.

9. Wishart GC, Loh SW, Jones L, Benson JR. A feasibility study (ICG-10) of indocyanine green (ICG) fluorescence mapping for sentinel lymph node detection in early breast cancer. Eur J Surg Oncol. 2012;38(8): 651-656.

10. Hirche C, Murawa D, Mohr Z, Kneif S, Hünerbein M. ICG fluorescenceguided sentinel node biopsy for axillary nodal staging in breast cancer. Breast Cancer Res Treat. 2010;121(2):373-378.

11. Ahmed M, Purushotham AD, Douek M. Novel techniques for sentinel lymph node biopsy in breast cancer: a systematic review. Lancet Oncol. 2014;15(8):e351-e362.

12. Livingston SF, Arlen M. The extended extrapleural radical mastectomy: its role in the treatment of carcinoma of the breast. Ann Surg. 1974; 179(3):260-265.
OncoTargets and Therapy

\section{Publish your work in this journal}

OncoTargets and Therapy is an international, peer-reviewed, open access journal focusing on the pathological basis of all cancers, potential targets for therapy and treatment protocols employed to improve the management of cancer patients. The journal also focuses on the impact of management programs and new therapeutic agents and protocols on

\section{Dovepress}

patient perspectives such as quality of life, adherence and satisfaction The manuscript management system is completely online and includes a very quick and fair peer-review system, which is all easy to use. Visit http://www.dovepress.com/testimonials.php to read real quotes from published authors. 